\title{
Extreme idealism and equilibrium in the Hotelling-Downs model of political competition
}

\author{
David Ronayne ${ }^{1}$ (D)
}

Received: 7 September 2017 / Accepted: 5 May 2018 / Published online: 23 May 2018

(C) The Author(s) 2018

\begin{abstract}
In the classic Hotelling-Downs model of political competition, no pure strategy equilibrium with three or more strategic candidates exists when the distribution of voters' preferred policies is unimodal. I study the effect of introducing two idealist candidates to the model who are non-strategic (i.e., fixed to their policy platforms), while allowing for an unlimited number of strategic candidates. Doing so, I show that equilibrium is restored for a non-degenerate set of unimodal distributions. In addition, the equilibria have the following features: (1) the left-most and right-most candidates (i.e., extremists) are idealists; (2) strategic candidates never share their policy platforms, which instead are spread out across the policy space; and (3) if more than one strategic candidate enters, the distribution of voter preferences must be asymmetric. I also show that equilibria can accommodate idealist fringes of candidates toward the extremes of the political spectrum.
\end{abstract}

Keywords Hotelling-Downs $\cdot$ Political competition $\cdot$ Equilibrium existence $\cdot$ Idealism

JEL Classification $\quad \mathrm{C} 72 \cdot \mathrm{D} 72$

I thank Dan Bernhardt, Peter Buisseret, Meg Meyer, Martin Osborne, Dan Quigley and Rob Schub for helpful discussion and comments. I also thank the Editor and two anonymous referees for their comments and suggestions.

Electronic supplementary material The online version of this article (https://doi.org/10.1007/s1112 7-018-0556-y) contains supplementary material, which is available to authorized users.

David Ronayne

david.ronayne@economics.ox.ac.uk

1 Department of Economics and Nuffield College, University of Oxford, 1 New Road,

Oxford OX1 1NF, UK 


\section{Introduction}

The Hotelling-Downs model of political competition is the workhorse of political scientists and political economists. A classical result is that candidates' incentives to maximize their vote shares will lead them to converge on the median voter's preferred platform in the unique equilibrium with $N=2$ candidates. In the model, strategic candidates do not choose extreme positions because it would render them unelectable. Furthermore, Osborne (1993) shows that, in general, when $N>2$, equilibria do not exist. However, many political races feature multiple candidates with distinct policy positions, i.e., distinct, extreme (left-most and right-most) candidates often run for public office. Candidates near the ends of the political spectrum compete at the polls in many countries, even when they are unlikely to win. Recent research suggests that the presence of extreme candidates within more mainstream parties may be due in significant part to candidates' own convictions (Bartels 2016). Using US data, Bartels shows a surprising lack of responsiveness of candidates' positioning to the views of swing voters. ${ }^{1}$ Rather than rejecting the workhorse model, in this article I investigate the impact of introducing idealist candidates into the baseline framework. Specifically, I suppose that in addition to the usual strategic candidates, two idealist candidates enter electoral contest who are fixed to their policy platforms. My first constructive result establishes that for equilibria to exist (within the class of unimodal distributions), such idealists must indeed be extremists, i.e., occupy left-most and right-most positions.

Osborne (1993) shows the negative result that the workhorse model, allowing for endogenous entry with $N>2$ strategic candidates who maximize their pluralities, fails to admit an equilibrium in pure strategies for all distributions of voter ideal points except for some pathological cases. Those cases constitute a degenerate class of distributions, including the uniform, which significantly weaken the results of previous studies that employed such distributions e.g., Cox (1987, Theorem 2). In this article, I show that for unimodal distributions of voter ideal points, no equilibria with more than two strategic entrants exist, but that the introduction of idealist candidates restores the existence of pure strategy equilibria for a non-degenerate set of unimodal distributions, and provide a characterization thereof. ${ }^{2}$ Moreover, I do so in a setting where the number of potential entrants is unlimited, i.e., $N=\infty$.

Platform-sharing is not an attractive prediction for empiricists. The second result I present says that in almost any equilibrium, it must be that exactly one strategic candidate positions himself or herself on any occupied policy platform. Combined with the fact that strategic candidates who enter tie, the result implies that their positions are spaced evenly throughout the distribution of voter preferences. This maximal differentiation of candidate positions in equilibrium shows that in such a setup, the hypothesis of platform-sharing is rejected strongly.

For symmetric distributions of voter ideal points, I find a unique equilibrium in which one strategic candidate enters and wins the election outright, when the idealists are not too extreme or too moderate relative to the distribution of voter preferences. I then show that if an equilibrium features multiple strategic entrants, then the distribution of voter ideal points is asymmetric, but that the converse is not true. I next provide a characterization

\footnotetext{
1 He also shows that the data are not consistent with the hypothesis that candidate positions are compromises between the locations of the relevant party's base and swing voters because candidates' positions tend to be even more extreme than the base.

${ }^{2}$ I study pure equilibria in this article and henceforth refer to them simply as "equilibria".
} 
of equilibria under asymmetric unimodal distributions. I also give examples of equilibria for various symmetric and asymmetric distributions. The main analysis is done with two idealist candidates. In the final section, I illustrate that equilibria are robust under the more general assumption that both ends of the political spectrum are populated with multiple idealist candidates, or "idealist fringes".

The paper proceeds as follows: Sect. 2 reviews some relevant literature; Sect. 3 presents the model; Sect. 4 provides and discusses the main results; Sect. 5 introduces idealist fringes; Sect. 6 concludes.

\section{Literature}

Many other researchers also have proposed variations of the canonical model in which a pure strategy equilibrium obtains when $N>2 .{ }^{3}$ Palfrey (1984) studies $N=3$ when two incumbent candidates choose their locations first, followed by a third-party candidate, and shows that the incumbents locate at distinct points in order to keep the entrant at bay (although the analysis quickly becomes intractable for larger $N$, see his Remark 1). Osborne (1993) defines a dynamic version of the model, and offers results for $N=3$ (and partial results for $N=4,5$ ) showing, among other findings, that an equilibrium always exists in which $N-2$ candidates enter and locate at the median. Equilibrium existence results have been perhaps more forthcoming in studies of electoral systems other than the one-vote, first-past-the-post setup. Xefteris (2016) shows that when one allows each voter to cast $k \geq 2$ votes, instead of just $k=1$, then equilibrium exists for a non-degenerate class of distributions when at least $k+1$ candidates enter at every location. Under runoff voting, Brusco et al. (2012) show that when $N>2$, equilibria (typically many) exist. In contrast, I offer results in a static setting with a plurality voting system (common to many countries, e.g., the United States, Canada, India and the United Kingdom) while allowing endogenous entry by an unlimited number of potential strategic candidates, i.e., $N=\infty$. $^{4}$

My model assumes the existence of idealistic candidates, not specifying the origin of their convictions. ${ }^{5}$ One approach that shares the feature of candidates being fixed to their platforms envisages them as members of the electorate who are assumed to be committed to imposing their own ideological stances on the polity, termed "citizen-candidates" (Besley and Coate 1997; Osborne and Slivinski 1996). Those approaches undoubtedly are deeper than the analysis in the baseline Hotelling-Downs model, insofar as candidates' origins are endogenous. Another related approach is the differentiated candidates framework of Krasa and Polborn $(2012,2014)$ which also allows for a multi-dimensional policy space, but assumes that the positions of two candidates are fixed in some dimensions, while flexible in others. In a setup with two strategic candidates and one extremist candidate, fixed to an end-point of the policy interval, Indridason (2013) compares equilibria across electoral systems. Xefteris et al. (2017) introduce centrifugal incentives in a general way to the classic paradigm with two strategic candidates, and show that their framework can be applied to study equilibrium in the presence of two extremist candidates (see their "Section A.2"). In contrast, the model of this article sticks closely to the canonical framework which in turn implies a reduction in richness. However, my model does incorporate the coexistence

\footnotetext{
3 For a survey of results with $N=2$, see Grofman (2004).

${ }^{4}$ Note that Xefteris (2016) also allows for $N=\infty$.

5 This assumption mirrors the agnosticism of Bartels (2016) as to the causes of candidates' convictions.
} 
of both ideological candidates (who stick to their positions) and an unlimited number of potentially strategic candidates (who could be interpreted as career politicians, with the sole goal of gaining office), endogenizing the entry decision of the latter type. The model also offers features that are consistent with some basic observations concerning elections, e.g., platform differentiation, multiple candidates and asymmetric distributions of voter preferences.

\section{Model}

The model's setup stays close to the canonical Hotelling-Downs model, generalizing it by allowing for endogenous entry, an unlimited number of candidates, and an objective function for strategic candidates as found in Osborne (1993). The policy space is represented by some interval $X \subseteq \mathbb{R}$. The ideal policies of voters are arrayed as described by an atomless distribution function $F$ with a continuous, unimodal pdf $f$ over support $X{ }^{6}$ Voters are assumed to be sincere and to have ideal points, meaning that they vote for the candidate positioned closest to that point. ${ }^{7}$ If multiple candidates locate at the same position, each of them receives an equal share of the votes from the voters for whom that position is closest. An unlimited number of strategic candidates (i.e., $N=\infty$ ) compete for votes, along with two idealist candidates. Idealist candidates always enter and occupy positions denoted by $z_{1}, z_{2} \in X$, where $z_{1}<z_{2}{ }^{8}$ Strategic candidates each have an action set $X \cup\{$ out , i.e., they either enter and choose a policy platform, or they choose not to enter the race. The number of strategic candidates choosing to enter the race is denoted $n$ and the vector of positions chosen, $x$. Candidates who do not enter are referred to as inactive. The functions $v_{i}: X^{n} \rightarrow[0,1]$ represent the vote share obtained by each candidate $i$ given a vector of positions $x$.

Regarding idealist candidates, the model is relatively agnostic as to the nature of their motivations. Idealism could stem from the convictions of the candidates themselves, or the convictions of any individual(s) controlling the candidate, e.g., the party's elite. What matters for the analysis is that idealist candidates are fixed to their positions, not who imposes the ideology upon the candidate. More generally, the model requires that the motivations of idealists, whatever they are, do not interact with the existence or locations of other candidates.

Strategic candidates maximize their pluralities, i.e., their margins of victory. Their preferences are represented by the following utility function:

$$
u_{i}(x)=v_{i}(x)-\max _{l \neq i}\left\{v_{l}(x)\right\}
$$

\footnotetext{
${ }^{6}$ Note that no restriction is imposed on whether $X$ is bounded (e.g., $X=[0,1]$ ) or unbounded (e.g., $X=(-\infty, \infty))$. An unbounded $X$ could be appropriate when no conceivable left-most and right-most policy position is possible (because e.g., it may always plausible to think of one "twice as extreme", and that some voter will have an ideal point there). Allowing for that possibility also enables commonly used distributions with unbounded supports to be analyzed, e.g., the normal distribution.

${ }^{7}$ Because $F$ is atomless, for voters with an ideal point such that they are indifferent between candidates at two distinct locations, it does not matter which candidate they choose (because the mass of such voters is zero).

${ }^{8}$ Although $z_{1}$ and $z_{2}$ refer to locations, sometimes I also call the idealists $z_{1}$ and $z_{2}$.
} 
An oft-used objective function for candidates is that of vote maximization. However, vote maximization is not a reasonable objective function for candidates when $N>2$, as it is incompatible with winning an election being preferred to losing it (Osborne 1995, p.280). To illustrate, I offer the following example: $X=[0,1], f$ uniform and position vector $x_{A}=(0,0.5,0.8)$, which gives $v_{1}\left(x_{A}\right)=0.25, v_{2}\left(x_{A}\right)=0.4, v_{3}\left(x_{A}\right)=0.35$, and hence a victory for candidate 2 . Now consider $x_{B}=(0,0.2,0.8)$, i.e., candidate 2 moves left, which gives $v_{1}\left(x_{B}\right)=0.1, v_{2}\left(x_{B}\right)=0.4, v_{3}\left(x_{B}\right)=0.5$ and a victory for candidate 3 . Under votemaximization, candidate 2 should be indifferent between $x_{A}$ and $x_{B}$ yet wins the election under $x_{A}$ and loses under $x_{B}$. Plurality maximization does not suffer from that criticism, saying that candidates prefer to: (1) win (or tie for the win) than to lose; (2) stay out than lose; and (3) win outright by wider margins. In addition, it is assumed that candidates prefer to win (outright or tie) than \{out\}, but prefer \{out $\}$ to entering and losing.

The occupied positions are denoted $y_{0}, \ldots, y_{r}$, indexed without loss of generality such that $y_{0}<\cdots<y_{r}$. The midpoint of two locations $y_{j}$ and $y_{j+1}$ is denoted $m_{j}=\frac{1}{2}\left(y_{j}+y_{j+1}\right)$. The total number of candidates located at $y_{j}$ is denoted $k_{j}$ (regardless of whether the candidates are idealistic or strategic). The constituency of a position $y_{j}$ is the share of voters that vote for one of the candidates at $y_{j}$. The left (right) constituency of $y_{j}$ denotes the mass of voters voting for a candidate at $y_{j}$ who have ideal points to the left (right) of $y_{j}$, denoted $L_{j}$, $R_{j}$ i.e., $L_{j}=F\left(y_{j}\right)-F\left(m_{j-1}\right)$ and $R_{j}=F\left(m_{j}\right)-F\left(y_{j}\right)$ for $j=0, \ldots, r$ where $F\left(m_{-1}\right) \equiv 0$ and $F\left(m_{r}\right) \equiv 1$.

\section{Results}

To motivate the positive results in the rest of the paper, I first reproduce the negative result of Osborne (1993) in the absence of idealist candidates, but applied to the class of unimodal densities of voter ideal points considered in this paper. There, I show that no equilibria with $n>2$ strategic entrants exist. Following this, I present necessary conditions for an equilibrium to exist in the presence of idealist candidates for almost any unimodal density $f{ }^{9}$ Those conditions include: idealists must be the extreme candidates and platforms are not shared (Propositions 2 and 3). I then add sufficient conditions in order to characterize equilibria for symmetric and asymmetric unimodal distributions (Propositions 4 and 5). Proofs, intermediate lemmas and longer expressions are relegated to the Online Supplementary Materials.

Proposition 1 (Without idealists). For any unimodel density $f$, without idealist candidates, no equilibrium with $n>2$ exists.

In analysis with strategic candidates only, conditions necessary for equilibrium are so restrictive that they preclude any equilibrium. In short, the problem stems from having strategic candidates at extreme locations. Here, I outline how in more detail. In equilibrium, an extreme position, let us say $y_{0}$, cannot be held by only one candidate, because if so, that candidate would have a strict incentive to move slightly towards $y_{1}$, increasing his or her vote share. Therefore, two candidates must be at $y_{0} \cdot{ }^{10}$ But then, it must be that

\footnotetext{
9 For details on what is meant by "almost any", see Lemma A4 in the Online Supplementary Materials.

10 There cannot be more than two, otherwise one of them could deviate profitably slightly to the left (or right).
} 
$L_{0}=R_{0}$ because otherwise, say if $L_{0}>R_{0}$, one of them could deviate profitably slightly to the left. When $n=2, y_{0}$ is the only location and $L_{0}=R_{0}$ is satisfied straightforwardly when $y_{0}=F^{-1}(1 / 2)$, i.e., when both locate at the median, which is the unique equilibrium and classic result of the Hotelling-Downs model. However, for an equilibrium with $n>2, y_{0}$ cannot be the only position. In this case, the requirement $L_{0}=R_{0}$ imposes requirements not only on where $y_{0}$ must be (in terms of $F$ ) but also where all remaining positions must be, relative both to one another and to $F$ itself. Those restrictions prove onerous, and prevent an equilibrium existing.

Proposition 2 (Extreme idealism). For almost any unimodal $f: y_{0}=z_{1}, y_{r}=z_{2}$ and $k_{0}=k_{r}=1$ in equilibrium.

Proposition 2 reveals that the left-most and right-most (i.e., extreme) positions must be occupied by idealists for an equilibrium to exist for almost any unimodal $f$. In the analysis discussed above with only strategic candidates, one necessary condition for an equilibrium is that two candidates must be at each extreme position $y_{0}$ and $y_{r}$. When combined with the other conditions, Proposition 1 shows that an equilibrium does not exist for $n>2$. That condition was a direct result of the incentives of strategic candidates: they will shift their platforms in order to increase their pluralities. However, when that strategic incentive is removed, e.g., because they are idealistically fixed to their platforms, the condition no longer is necessary. As a result, the restrictive conditions on $F$, found in the case when strategic candidates must occupy the extreme positions, are not incurred. That allows me to show that for equilibrium to exist generically, extreme positions must be occupied by candidates without strategic concerns, i.e., Proposition 2: in equilibrium, extremists must be idealists.

Proposition 3 (No platform sharing). For almost any unimodal $f, k_{j}=1$ for all $j$ when $n \geq 2$ in equilibrium.

Proposition 2 dealt with the extreme locations. Proposition 3 deals with the intermediate positions and shows that they also cannot generally hold more than one strategic candidate in equilibrium. Owing to the endogenous entry decision, all strategic candidates who enter tie in equilibrium. Together with Proposition 3, that result implies that a necessary equilibrium condition is that the strategic candidates are spaced evenly throughout the distribution of voter preferences. In contrast, the classic convergence hypothesis stipulates that candidates are incentivized to converge on shared locations. The separation of candidates' equilibrium positions here shows that the hypothesis can fail, irrespective of the number of strategic entrants. Furthermore, the fact that strategic candidates are spaced evenly across the voter distribution departs markedly from convergence. In the sense that each strategic candidate is as far from another as possible, one could interpret the result as implying maximal divergence. In the classic Hotelling-Downs setup with $n=2$, the only force is centripetal: candidates want to move closer to one another. That is because with only one other candidate, moving towards that candidate comes at no loss. However, when more than two candidates are running, a candidate can find himself or herself between two others. In that scenario, moving towards one of the other candidates comes at the loss of votes to the candidate from whom one moves away. Having dealt with the problem (Proposition 1), which resulted from having strategic types at the extreme positions (Proposition 2), a balancing of 

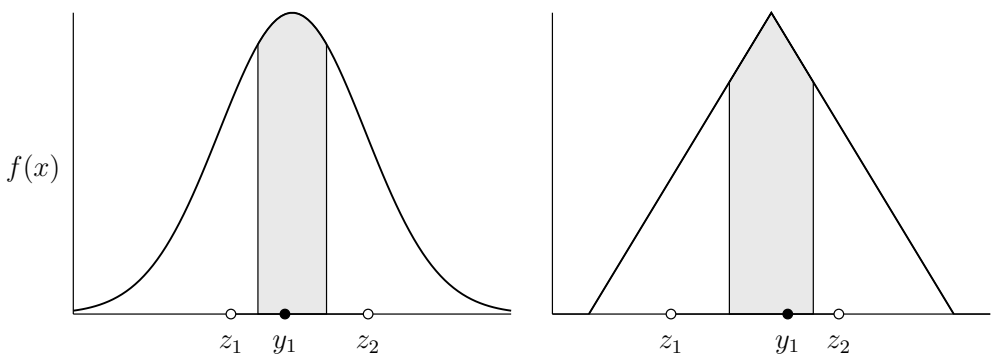

Fig. 1 Equilibrium for symmetric unimodal distributions. Left panel: Differentiable $f$ with unbounded support (the standard normal); idealists at percentiles 20 and 85 . One strategic candidate enters at $y_{1}$, which is percentile 46 , and obtains a vote share of 0.36 . The idealists' vote shares both are 0.32 . Right panel: Non-differentiable $f$ with bounded support (a triangular distribution); idealists at percentiles 10 and 80 . One strategic candidate enters at $y_{1}$ which is percentile 59, and obtains a vote share of 0.41 . The idealists' vote shares are both 0.30 . Hollow (filled) circles represent the locations of idealist (strategic) candidates. Shaded (unshaded) areas are the constituencies of the winning (losing) candidate

those forces is what leads to Proposition 3. One difference with many of the divergence results in the literature is that candidates face no explicit centrifugal incentives. Rather, multiple competing centripetal forces exist: incentives to move towards each of your neighbors are equally powerful (Proposition 2 ensures that each strategic candidate has a neighbor on both sides). Those competing forces must be balanced in equilibrium, which is why strategic candidates must be spread evenly throughout the policy space.

The results of Propositions 2 and 3 lay the groundwork for the equilibrium characterizations. Proposition 4 provides the conditions for which a unique equilibrium for symmetric unimodal distributions of voter ideal points exists.

Proposition 4 (Symmetric distributions). For almost any symmetric, unimodal $f, a$ unique equilibrium exists wherein $n=1$ strategic candidate enters at location $y_{1}$, where $y_{1}$ solves (1):

$$
F\left(m_{0}\right)=1-F\left(m_{1}\right)
$$

and the positions of the idealists $\left(z_{1}, z_{2}\right)$ satisfy (2) and (3):

not too moderate: $m_{0}<F^{-1}\left(\frac{1}{3}\right) \Longleftrightarrow m_{1}>F^{-1}\left(\frac{2}{3}\right)$;

not too extreme: if $z_{1}$ is closer to the maximizer of $f$ than $z_{2}$ is, $F\left(y_{1}\right) \geq 1-2 F\left(m_{0}\right)$;

if $z_{2}$ is closer to the maximizer of $f$ than $z_{1}$ is, $F\left(y_{1}\right) \leq 2 F\left(m_{0}\right)$.

Except for unimodality, the conditions of Proposition 4 deliver equilibrium existence without any other restrictions on the shape of $f$. Condition (1) is implied by the requirement that the idealists' vote shares must be equal in equilibrium (if not, then, owing to symmetry, the strategic candidate could deviate profitably by moving slightly towards the idealist with the larger vote share). Conditions (2)-(3) state that, relative to the distribution of voter preferences, the idealists cannot be too moderate or too extreme. They cannot be too moderate because a strategic candidate must win (specifically, their constituencies must 
be clear of the central third of $F$ ). They cannot be too extreme, else an inactive candidate is given room within which to enter and win. I now illustrate the characterization with two examples, depicted in Fig. 1.

Example 1 Let $F$ be the standard normal distribution and the idealists be located at percentiles 20 and 85: $\left(z_{1}, z_{2}\right)=\left(F^{-1}(0.20), F^{-1}(0.85)\right)=(-0.84,1.04)$. Condition (1) then gives $y_{1}=-0.10$. The remaining conditions also are satisfied: (2) becomes $m_{0}=-0.47<-0.43=F^{-1}(1 / 3)$ and the first statement of (3) becomes $F\left(y_{1}\right)=0.46 \geq 0.36=1-2 F\left(m_{0}\right)$. The left panel of Fig. 1 shows this equilibrium.

Example 2 Let $F$ be the triangular distribution with the density $f(x)=1-|x|$ for $x \in[-1,1]$ and the idealists be located at percentiles 10 and 80: $\left(z_{1}, z_{2}\right)=\left(F^{-1}(0.1), F^{-1}(0.8)\right)=(-0.55,0.37)$. Condition $(1)$ then gives $y_{1}=0.09$. The remaining conditions also are satisfied: (2) becomes $m_{0}=-0.30<-0.18=F^{-1}(1 / 3)$ and the second statement of (3) becomes $F\left(y_{1}\right)=0.73 \geq 0.51=1-2 F\left(m_{0}\right)$. The right panel of Fig. 1 shows this equilibrium.

In Fig. 1, we can now see the balancing of forces required of strategic entrants in equilibrium most clearly. The strategic candidate would like to win the votes at either margin of their constituency, all else equal. However, he or she has a candidate on each side and therefore cannot always shift closer to one of them without losing votes to the other. The candidates without a neighbor on both sides are idealists and, hence, do not move closer to their neighbors. Finally, the exact equilibrium location of the strategic candidate is pinned down by the point that maximizes his or her plurality. ${ }^{11}$ In the case of symmetric distributions, that is where the density at the two boundaries of the constituency is equal.

A feature of Proposition 4 is that with symmetric unimodal densities, only one strategic candidate enters in equilibrium. In Corollary 1, I show that this feature is not special to symmetry per se: it will hold in equilibrium for almost any unimodal distribution for which the mode (Mo) equals the median (Md).

\section{Corollary 1. For almost any unimodal $f$ where $\operatorname{Mo}(f)=\operatorname{Md}(f), n=1$.}

To understand the result, suppose instead that $n>1$, implying that exactly one idealist loses (see Lemma A7 in the Online Supplementary Materials). Furthermore, no more than one strategic candidate with any of his or her constituency on the same side of the mode as the losing idealist is possible (else the candidate closest to the losing idealist could deviate profitably by moving slightly towards the mode). There then must be at least one strategic candidate with his or her whole constituency on the same side of the mode as the idealist who ties for the win. However, for those candidates to win, more than half of the probability density must be on that side of the mode, contradicting $\operatorname{Mo}(f)=\operatorname{Md}(f)$.

I now characterize equilibria with $n>1$ strategic entrants. By Corollary 1 we know that distributions of voter preferences supporting such equilibria are such that $\operatorname{Mo}(f) \neq \operatorname{Md}(f)$ and, hence, are asymmetric. Furthermore, the simple fact of the median or the mode of

\footnotetext{
11 Note that plurality maximization generates a unique equilibrium prediction. If instead, a simple win motivation was assumed for strategic candidates (so that they are indifferent between wins of all sizes), then a continuum of equilibrium locations would exist, e.g., in Example 1, any $y_{1} \in[-0.32,0.12]$ would be an equilibrium.
} 
$f$ being greater will play a role in determining equilibria. Proposition 5 provides conditions for an equilibrium to exist for asymmetric unimodal distributions of voter preferences when $\operatorname{Mo}(f) \neq \operatorname{Md}(f)$. Proposition 2 gives two examples.

Proposition 5 (Asymmetric distributions). For almost any asymmetric, unimodal $f$ satisfying (4)-(6) where $\operatorname{Mo}(f) \neq \operatorname{Md}(f)$, an equilibrium exists with $n>1$ strategic candidates where locations and vote-shares are given by Lemma A10 in the Online Supplementary Materials.

$$
\begin{array}{ll}
\text { If } \operatorname{Mo}(f)<\operatorname{Md}(f) & \text { If } \operatorname{Mo}(f)>\operatorname{Md}(f) \\
f\left(m_{0}\right) \in\left[f\left(m_{1}\right), 2 f\left(m_{1}\right)\right] & f\left(m_{n}\right) \in\left[f\left(m_{n-1}\right), 2 f\left(m_{n-1}\right)\right] \\
f\left(m_{j-1}\right) \leq 2 f\left(m_{j}\right) \quad j=2, \ldots, n & f\left(m_{j}\right) \leq 2 f\left(m_{j-1}\right) \quad j=1, \ldots, n-1 \\
f\left(m_{0}\right) \leq \max \left\{f\left(y_{1}\right), f\left(z_{1}\right)\right\} & f\left(m_{n}\right) \leq \max \left\{f\left(y_{n}\right), f\left(z_{2}\right)\right\}
\end{array}
$$

Compared to the symmetric case, additional equilibrium conditions are necessary when $n>1$. Lemma A10 provides conditions (A6) and (A9), which are analogous to condition (2) of Proposition 4, saying that the losing idealist must be extreme enough to lose. The Lemma also provides the exact equilibrium locations of strategic candidates (conditions A4, A5, A7, A8), which as Proposition 3 revealed, are spaced evenly throughout the distribution of voter ideal points. Specifically, the locations of the idealist candidates pin down the vote share, $s^{*}$, enjoyed by each of the strategic candidates in equilibrium. The strategic candidates' locations are then determined by a "spacing procedure" (detailed in precisely in Lemma A8). To illustrate, suppose that $z_{1}$ ties for the win (which is the case if $\operatorname{Mo}(f)<\operatorname{Md}(f))$; then place the first strategic candidate at $y_{1}$, such that $z_{1}$ has a vote share of $s^{*}$; then place the second strategic candidate at $y_{2}$, such that the candidate at $y_{1}$ has a vote share of $s^{*}$, and so on; the losing idealist, in this case $z_{2}$, will then be left with the residual vote share of $1-s^{*}(n+1)$.

For equilibria with $n>1$, conditions concerning the shape of $f$ are imposed, given by (4)-(6). The requirement of (4) and (5) that $f\left(m_{j-1}\right) \leq 2 f\left(m_{j}\right)$ for $j=1, \ldots, n$ is driven by the fact that strategic candidates are plurality maximizers. Condition (6) requires that the density of the midpoint between the losing extremist and the neighboring strategic candidate not be higher than the density of both of those candidates' locations. The condition precludes the possibility of a profitable entry by an inactive candidate. All conditions are met by the examples in Fig. 2, which therefore are equilibria with asymmetric, unimodal distributions of voter preferences.

The intuition of the conditions and earlier results can be seen in Fig. 2. As was the case for equilibrium with symmetric distributions, strategic candidates must have balanced their incentives to move towards each of their neighbors. That balance manifests in a slightly different way than with $n=1$. Take the example in the top panel of Fig. 2 and the candidate at $y_{1}$. Consider why they do not wish to move slightly left. Doing so would gain $f\left(m_{0}\right)$ votes, but would lose $f\left(m_{1}\right)$ votes to the candidate at $y_{2}$, who then would have the highest vote share of all the others, meaning that it is that candidate who determines $y_{1}$ 's plurality (this consideration was absent under symmetry when $n=1$ because there the candidates at $y_{1}$ and $y_{2}$ did not tie). In sum, a move to the left would result in a 


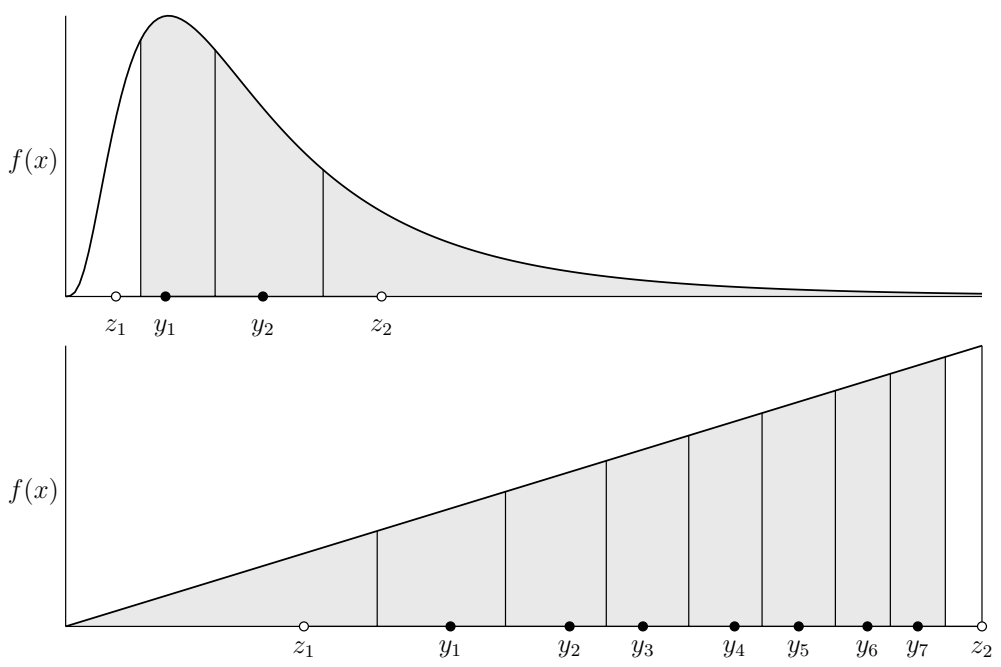

Fig. 2 Equilibrium for asymmetric unimodal distributions with $n>1$ strategic candidates. Top panel: $f$ is the $\log$-normal distribution $\ln \mathcal{N}(0,0.5)$; idealists at percentiles 5 and 80 . Two strategic candidates enter at $y_{1}$ and $y_{2}$, which are percentiles 23 and 58, respectively, and both obtain a vote share of 0.29 . The idealists $z_{1}$ and $z_{2}$ obtain vote shares of 0.13 and 0.29 , respectively. Bottom panel: $f$ is the linear distribution; idealists at percentiles 7 and 100 . Seven strategic candidates enter at $y_{1}, \ldots, y_{7}$, which, respectively, are percentiles $(17,30,40,53,63,77,86)$, and all obtain a vote share of 0.116 . The idealists $z_{1}$ and $z_{2}$ obtain vote shares of 0.116 and 0.07 respectively. Hollow (filled) circles represent the locations of idealist (strategic) candidates. Shaded (unshaded) areas are the constituencies of the winning (losing) candidates

change of plurality equal to $f\left(m_{0}\right)-2 f\left(m_{1}\right)$. That change is not positive if $f\left(m_{0}\right) \leq 2 f\left(m_{1}\right)$, which is satisfied in the example. No profitable deviation for this candidate slightly to the right exists because doing so would gain $f\left(m_{1}\right)$ but lose $f\left(m_{0}\right)$ to $z_{1}$. However, for a small move, $z_{1}$ still loses, and so does not determine $y_{1}$ 's plurality directly. Therefore, the move to the right would result in a change of plurality equal to $f\left(m_{1}\right)-f\left(m_{0}\right)$. That move is not profitable if $f\left(m_{1}\right) \leq f\left(m_{0}\right)$, which is satisfied in the example. As for the candidate at $y_{2}$, local deviations in both directions cause the neighbor they move away from to win, which, by following the first case above for the candidate at $y_{1}$ results in the requirements that $f\left(m_{1}\right) \leq 2 f\left(m_{2}\right)$ and $f\left(m_{2}\right) \leq 2 f\left(m_{1}\right)$. However, because of the unimodality of $f$, the second condition is implied automatically (because the whole of $y_{2}$ 's constituency is to the right of the mode, $\left.f\left(m_{2}\right)<f\left(m_{1}\right)\right)$. As in the symmetric case, it suffices to consider deviations of this sort for candidates with neighbors on both sides, because those with neighbors on one side are idealists who do not move.

In reality, many elections are held with a small number of main parties (generally more than two, but often exactly two) who could be considered to be strategic actors. Here, I explore in more intuitive terms how the formal statements of Proposition 5 can provide conditions regarding when equilibria predict fewer strategic entrants. To do so, I take the equilibrium with $n=2$ shown in the top panel of Fig. 2 as a running example. Firstly, I consider the impact of changes in the steepness of $f$. In Fig. 2, the distribution of voters was sufficiently flat in the necessary places such that $f\left(m_{1}\right)<2 f\left(m_{2}\right)$, i.e., (5), was satisfied. However, if we consider changing $f$ such that $f$ is steeper throughout the constituency of $y_{2}$, 
then at some point $f\left(m_{1}\right)>2 f\left(m_{2}\right)$ and, therefore, equilibrium with $n=2$ breaks down. In that way, we can remark that in order to have equilibria with few strategic candidates, the distribution of voter preferences cannot be too steep in too many places. Secondly, consider the position of the idealists, specifically the idealist who ties $\left(z_{2}\right.$ in the example). As $z_{2}$ becomes increasingly extreme, a larger proportion of that candidate's vote share comes from their left constituency. Because $f$ is unimodal and equilibrium requires the whole of $y_{2}$ 's constituency to be on the right of the mode of $f$, the equilibrium locations of the strategic candidates must converge in order for them to tie with $z_{2}$. However, at some point, $z_{2}$ becomes so extreme that either $y_{1}=y_{2}$ or $z_{1}$ wins, causing equilibrium with $n=2$ to break down. This logic shows that in order to have equilibria with few strategic candidates, a second requirement is that idealists are not too extreme.

The assumption of unimodality of $f$ provided a strong negative result (Proposition 1) and relatively succinct positive results (Propositions 4 and 5). I now discuss how one could relax the assumption of unimodality of the distribution of voter ideal points while maintaining the equilibria derived in Propositions 4 and 5. In short, such equilibria can still exist if unimodality is dropped, but many more equilibrium considerations are needed. Here, I describe the nature of some of those considerations. Firstly, note that any characterization will no longer be divided into cases by a comparison of mode versus median, because multiple modes may exist. Second, consider the density between the idealists, where all the strategic candidates are in equilibrium. ${ }^{12}$ Strategic candidates' positions will require conditions analogous to those listed in Propositions 4 and 5 in the cases of $n=1$ and $n>1$, respectively. Additionally, when $n>1$, if local modes are close enough to, and less extremely located than, both idealists, it can be that no idealist ties in equilibrium, which requires its own set of considerations. Also, when $f$ is unimodal, condition (6) ensured no profitable deviation of an inactive candidate into a constituency which contained the mode. However, when $f$ has many local modes, there need to be conditions in place such that there is no such profitable opportunity at any local mode. This could be achieved by there being a higher number of strategic entrants in equilibrium, such that each local mode has a candidate sufficiently close to it. However, whether that possibility is achievable depends itself on $F$ and the location of the idealists. It depends on $F$ because the local modes must be spaced throughout $F$ in such a way that the strategic entrants all can tie. It depends on the idealists' positions because one cannot keep adding more strategic candidates ad infinitum in order to satisfy other considerations because at some point the density will be shared too thinly between them and an idealist will win outright, which cannot occur in any equilibrium with strategic entrants. Such complications do not arise when $f$ is unimodal because there is only one local mode to consider.

\section{Idealist fringes}

The preceding analysis assumed the existence of two idealist candidates which kept the analysis more tractable. However, the model can be extended beyond the two-idealist setup. Here, I show that equilibria can accommodate multiple idealist candidates at the extremes of the political spectrum, which I term "idealist fringes". Their introduction requires minor

\footnotetext{
12 Note that in any equilibrium it is unimportant what the density function looks like to the left of the leftextremist and to the right of the right-extremist: there is never enough density there in equilibrium to offer a profitable deviation to either active or inactive candidates, and so generalizing $f$ there comes without loss.
} 


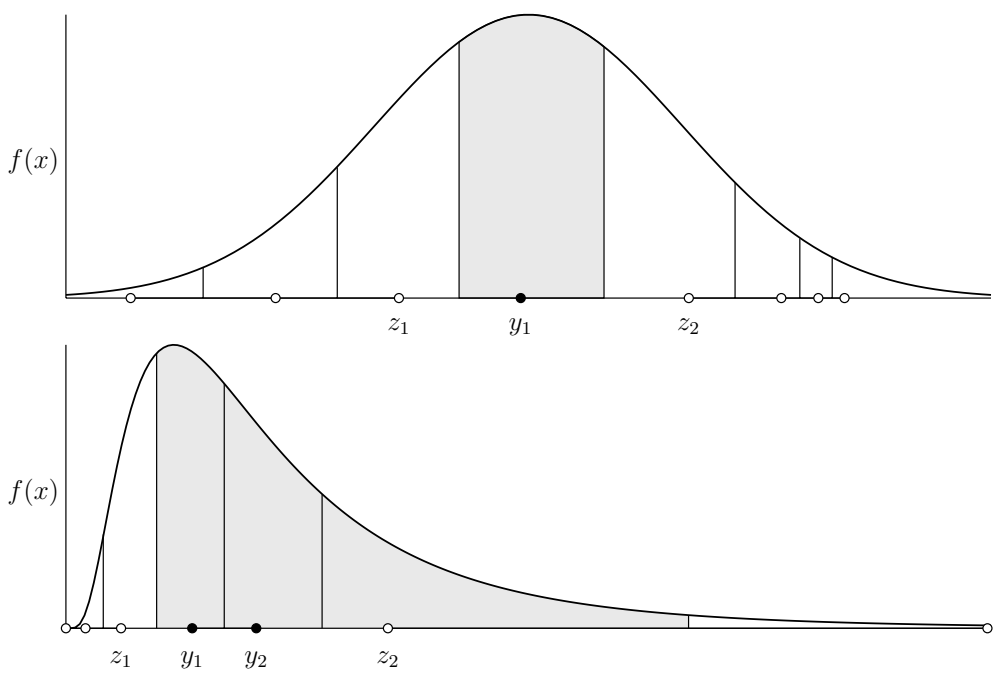

Fig. 3 Equilibrium with idealist fringes. Top panel: $f$ is the standard normal as in the left panel of Fig. 1; idealists at percentiles $0.5,5,20$ on the left and 85, 95, 97, 98 on the right. One strategic candidate enters at $y_{1}$, which is percentile 48 , and obtains a vote share of 0.36 . The idealists' vote shares, from left to right, respectively, are $(0.02,0.09,0.22,0.22,0.05,0.01,0.02)$. Bottom panel: $f$ is the lognormal distribution $\ln \mathcal{N}(0,0.5)$ as in the top panel of Fig. 2; idealists at percentiles $0,0.1,5$ on the left and 80,99 on the right. Two strategic candidates enter at $y_{1}$ and $y_{2}$, which are percentiles 31 and 54, respectively, and both obtain a vote share of 0.26 . The idealists' vote shares, from left to right, respectively, are $(<0.001,0.01,0.16,0.26,0.04)$. Hollow (filled) circles represent the locations of idealist (strategic) candidates. Unlabeled circles represent the idealists not included in the corresponding panels of Figs. 1 and 2. Shaded (unshaded) areas are the constituencies of the winning (losing) candidates

re-workings of the equilibrium conditions derived previously. In Fig. 3, I augment examples from Figs. 1, 2 to incorporate idealist fringes.

Here, I contrast the equilibria shown in Fig. 3 relative to the corresponding panels of Figs. 1 and 2. In the symmetric example where $f$ is the standard normal, the equilibrium location of strategic candidate $y_{1}$ is such that the adjacent idealists, $z_{1}$ and $z_{2}$, tie for second place. That is the analog of condition (1) of Proposition 4 and, similarly, ensures that the strategic candidate does not want to deviate within his or her constituency. Proposition 4's conditions (2) and (3) also are reflected, respectively, by the facts that the idealists are located such that the strategic candidate wins and that inactive strategic candidates prefer not to enter. Introducing any additional number of idealist candidates to the fringes such that they do not change the vote shares of $z_{1}$ or $z_{2}$ will not alter the equilibrium beyond changing the vote shares of those candidates in the fringes (e.g., the idealists shown at percentiles 0.5, 97 and 98). In the asymmetric example, the equilibrium locations of the strategic candidates are recalculated to ensure that $y_{1}, y_{2}$ and $z_{2}$ all tie for first place. A recalculation is necessary because the introduction of the idealist to the right of $z_{2}$ reduced $z_{2}$ 's constituency. The idealists introduced to the left of $z_{1}$ have no effect on the equilibrium other than changing the vote shares of the idealists in the left fringe (e.g., the idealists shown at percentiles 0 and 0.1). Similarly, introducing idealists to the right of the far-right idealist candidate likewise would have no effect on the equilibrium except altering the vote shares of candidates on the right fringe. The positions depicted also satisfy conditions (4)-(6) of Proposition 5 and so constitute an equilibrium. 


\section{Conclusion}

I analyzed a variant of the canonical Hotelling-Downs model which features idealist candidates in addition to the standard strategic candidates. In doing so, I found that equilibria exist generically within the class of unimodal distributions of voter ideal points, while allowing for an unlimited number of potential entrants. That result stands in contrast to the setting without idealists where no equilibria exist with more than two strategic entrants. The model makes a number of predictions. Those more straight-forward are that (for almost any unimodal distribution of voter preferences): (i) extreme candidates will tend to be ideologically fixed to their platforms and that (ii) strategic candidates locate on distinct policy platforms. Other predictions include a relationship between the mode and median of $f$ as a determinant of the number of candidates entering in equilibrium: If multiple strategic candidates enter, the distribution of voter preferences is such that the mode and median are distinct. Conversely, if the distribution of voter preferences is symmetric, one strategic candidate will run and win. A binary comparison between mode and median cannot of course capture all of the ways in which distributions can be asymmetric, but nevertheless acts as a succinct predictive measure in plurality voting systems with idealist candidates. Finally, I showed how equilibria can accommodate 'idealist fringes' when multiple idealistic candidates populate the extremes of the political spectrum.

The analysis speaks to several active discussions within the literature. Theoretically, its main contribution is to make the novel point that modeling idealist along with standard strategic candidates can help restore equilibrium. However, it also predicts a failure of the convergence hypothesis: all of the equilibria presented have the feature that strategic candidates spread evenly throughout the policy space. In my model, divergence occurs because of the need for strategic candidates to balance centripetal forces (the incentives to move towards each of their neighbors), rather than as a result of a centrifugal force. In work with two strategic candidates, divergence has been generated in other, related ways. Calvert (1985) showed that the convergence hypothesis is robust to candidates either being both win- and policy-motivated, or uncertain over voters' responses, but that in the presence of both assumptions, divergence is predicted. Peress (2010) introduces an asymmetric non-policy dimension to candidates, which allows the candidate stronger in that dimension to move away from the center towards his or her own preferred policy (also see Peress 2013, which empirically selects between theories of divergence). Waters (2017) models parties and candidates as separate entities, allowing parties to choose platforms and to "whip" candidates, which reduces voter uncertainty and leads to divergence.

Recent empirical work examines the presence and connections between candidates' extremism, idealism and electoral success. As noted in the Introduction, Bartels (2016) suggests that US presidential candidates' extreme positions may be explained in a large part by their own ideologies. Carson and Williamson (2017) find that when candidates in races for the US House of Representatives move towards platforms that are extreme relative to voters, they are less likely to win. As for multi-party races, Wagner and Meyer (2017) find support for the notion that although radical right parties in Europe have remained niche players, they have affected the positioning of mainstream parties across the spectrum. These findings chime with the core features of my model: that idealists tend to be extreme, to lose, and to affect the positioning of their competitors. Therefore, a broad group of settings seem to exist in which the model presented here is at least somewhat 
applicable. The model may also apply more naturally in settings when relatively extreme idealist candidates are more likely to enter races, e.g., jurisdictions where the cost of entry is lower or where it is easier for politically inexperienced individuals to run (see e.g., Carson and Williamson 2017). On the other hand, I also note that not all types of extreme idealism are covered by the model, e.g., when idealists are opposed to, and refuse to partake in, democratic processes, or are committed to expressing their views only through violence.

\section{Compliance with ethical standards}

Conflict of interest The author declares that he has no conflict of interest.

Open Access This article is distributed under the terms of the Creative Commons Attribution 4.0 International License (http://creativecommons.org/licenses/by/4.0/), which permits unrestricted use, distribution, and reproduction in any medium, provided you give appropriate credit to the original author(s) and the source, provide a link to the Creative Commons license, and indicate if changes were made.

\section{References}

Bartels, L. M. (2016). Failure to converge. Annals of the American Academy of Political and Social Science, 667(1), 143-165.

Besley, T., \& Coate, S. (1997). An economic model of representative democracy. The Quarterly Journal of Economics, 112(1), 85-114.

Brusco, S., Dziubiński, M., \& Roy, J. (2012). The Hotelling-Downs model with runoff voting. Games and Economic Behavior, 74(2), 447-469.

Calvert, R. L. (1985). Robustness of the multidimensional voting model: Candidate motivations, uncertainty, and convergence. American Journal of Political Science, 29(1), 69-95.

Carson, J. L., \& Williamson, R. D. (2017). Candidate ideology and electoral success in congressional elections. Public Choice. https://doi.org/10.1007/s11127-017-0492-2.

Cox, G. W. (1987). Electoral equilibrium under alternative voting institutions. American Journal of Political Science, 31(1), 82-108.

Downs, A. (1957). An Economic Theory of Democracy. New York: Harper \& Row.

Grofman, B. (2004). Downs and two-party convergence. Annual Review of Political Science, 7, $25-46$.

Hotelling, H. (1929). Stability in competition. Economic Journal, 3, 41-57.

Indridason, I. H. (2013). Expressive motives and third-party candidates. Journal of Theoretical Politics, 25(2), 182-213.

Krasa, S., \& Polborn, M. K. (2012). Political competition between differentiated candidates. Games and Economic Behavior, 76(1), 249-271.

Krasa, S., \& Polborn, M. K. (2014). Social ideology and taxes in a differentiated candidates framework. American Economic Review, 104(1), 308-322.

Osborne, M. J. (1993). Candidate positioning and entry in a political competition. Games and Economic Behavior, 5(1), 133-151.

Osborne, M. J. (1995). Spatial models of political competition under plurality rule: A survey of some explanations of the number of candidates and the positions they take. The Canadian Journal of Economics, 28(2), 261-301.

Osborne, M. J., \& Slivinski, A. (1996). A model of political competition with citizen-candidates. Quarterly Journal of Economics, 111(1), 65-96.

Palfrey, T. R. (1984). Spatial equilibrium with entry. Review of Economic Studies, 51(1), 139-156.

Peress, M. (2010). The spatial model with non-policy factors: A theory of policy-motivated candidates. Social Choice and Welfare, 34(2), 265-294.

Peress, M. (2013). Candidate positioning and responsiveness to constituent opinion in the US House of Representatives. Public Choice, 156(1-2), 77-94.

Wagner, M., \& Meyer, T. M. (2017). The radical right as niche parties? The ideological landscape of party systems in Western Europe, 1980-2014. Political Studies, 65, 84-107.

Waters, T. D. P. (2017). Cracking the whip: Spatial voting with party discipline and voter polarization. Public Choice, 173(1-2), 61-89. 
Xefteris, D. (2016). Stability in electoral competition: A case for multiple votes. Journal of Economic Theory, 161, 76-102.

Xefteris, D., Laussel, D., \& Le Breton, M. (2017). Simple centrifugal incentives in spatial competition. International Journal of Game Theory, 46(2), 357-381. 